\title{
Feasibility of field-based light scattering spectroscopy
}

\author{
Changhuei Yang \\ Lev T. Perelman \\ Adam Wax \\ Ramachandra R. Dasari \\ Michael S. Feld \\ G. R. Harrison Spectroscopy Laboratory \\ Massachusetts Institute of Technology, \\ Cambridge, Massachusetts 02139
}

\begin{abstract}
Light scattering spectroscopy (LSS) is a new technique capable of accurately measuring the features of nuclei and other cellular organelles in situ. We present the considerations required to implement and interpret field-based detection in LSS, where the scattered electric field is detected interferometrically, and demonstrate that the technique is experimentally feasible. A theoretical formalism for modeling field-based LSS signals based on Mie scattering is presented. Phase-front uniformity is shown to play an important and novel role. Results of heterodyne experiments with polystyrene microspheres that localize LSS signals to a region about $30 \mu \mathrm{m}$ in axial extent are reported. In addition, differences between field-based LSS and the earlier intensity-based LSS are discussed. () 2000 Society of Photo-Optical Instrumentation Engineers. [S1083-3668(00)00902-3]
\end{abstract}

Keywords: light scattering spectroscopy; interferometer; Mie theory.

Paper JBO-42014 received Sep. 15, 1999; revised manuscript received Feb. 8, 2000; accepted for publication Mar. 1, 2000.

\section{Introduction}

Recently, a new light scattering technique has been used during endoscopic procedures to measure the size distribution of cell nuclei and refractive index changes in the epithelial linings of the body. ${ }^{1-4}$ In these studies, the intensity of white light backscattered from the tissue is collected via an optical fiber probe and spectrally analyzed. The cell nuclei behave like Mie scatterers $;^{5}$ such particles exhibit periodic intensity variations with wavelength that are proportional to their sizes (typically 5-15 $\mu \mathrm{m}$ ) and relative refractive indices. This technique, called light scattering spectroscopy (LSS), is of interest because changes in the size of cell nuclei and their chromatin content (related to refractive index) are primary indicators of dysplasia, ${ }^{6}$ the precursor of cancer, and treatment is most simple and effective when implemented at this early stage. ${ }^{6}$

Until now, the LSS information has been obtained from analysis of the intensity of the backscattered light. In this article, we shall explore the feasibility of employing electric field based techniques to measure LSS signals, by applying interferometry to the measurement process. Field-based LSS with low-coherence light sources can isolate regions as small as $30 \mu \mathrm{m}$ in dimension for study (as limited by the coherence length of the light source). The ability of low coherence interferometry to localize small regions for measurements has been amply demonstrated in recent studies of optical coherence tomography (OCT). ${ }^{7-9}$ In addition, field-based measurements preferentially detect light which has been scattered only once, ${ }^{10}$ and greatly discriminate direct backscattering from diffuse tissue reflectance, thus enhancing sensitivity and simplifying the computations required to extract the size distribution of the scatterers.

A fully implemented field-based LSS system would measure the light scattering signals over a broad spectral range. From these measurements, we would be able to extract information about the nuclei size distributions and chromatin content of a few or even a single cell in vivo. In addition, the

Address all correspondence to Changhuei Yang. E-mail: chyang@mit.edu potential greater sensitivity of field-based LSS may allow for study of smaller organelles and extracellular structures. As a feasibility assessment of this technique and the first step in its implementation, we conducted a field-based LSS experiment using two wavelengths.

This article presents the considerations required to implement field-based LSS, and demonstrates its feasibility through an experimental study. A theoretical formulation based on light scattering theory for modeling and interpreting fieldbased LSS is presented. Phase-front uniformity is shown to play an important role. We report a proof-of-principle experiment that measures the LSS signals arising from microsphere scattering within a localized region $30 \mu \mathrm{m}$ in axial extent. In addition, differences between intensity- and field-based LSS methods are discussed.

\section{Experimental Method}

The experimental setup (Figure 1) employs a Michelson interferometer with two low-coherence light sources. A Coherent MIRA Ti: sapphire laser operating in femtosecond mode (150 fs) produces $800 \mathrm{~nm}$ light. A portion of this light is split off and up-converted to $400 \mathrm{~nm}$ by means of a CSK Optronics LBO second harmonic generation crystal. The coherence lengths of both the primary and second harmonic are about 30 $\mu \mathrm{m}$. The converted light is then recombined with the original beam. Care is taken to achieve good spatial overlap between the two wavelength components. The combined beam is then divided by a beam-splitter (BS1) into probe and reference beams.

The probe beam is focused onto the sample by means of a $12.7 \mathrm{~mm}$ focal length achromatic lens. The powers of the 400 and $800 \mathrm{~nm}$ components at the sample are 14 and $5.5 \mathrm{~mW}$, respectively. The beam waists at the lens are 1.1 and $2.1 \mathrm{~mm}$ [full width at half maximum (FWHM)], resulting in beam waists at the focal point of 5.9 and $6.2 \mu \mathrm{m}$, and Rayleigh lengths of 270 and $150 \mu \mathrm{m}$, respectively. As the coherence 


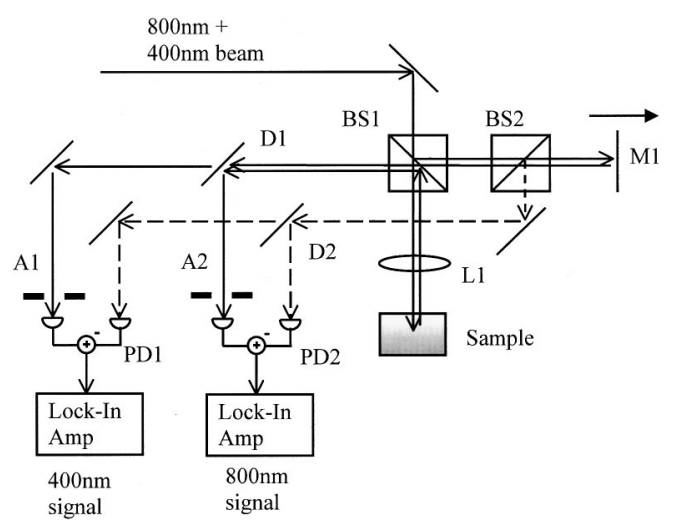

Fig. 1 Experimental setup.

length is shorter than the Rayleigh lengths, we can effectively approximate the probe region as a cylinder. The calculated beam overlap within this region is $98 \%$, insuring that the same particles within the sample are illuminated simultaneously at both wavelengths.

The reference beam is reflected from a mirror (M1) moving at a constant speed, inducing Doppler shifts of 14.6 and $7.3 \mathrm{kHz}$ at 400 and $800 \mathrm{~nm}$, respectively. It is then recombined with the probe light backscattered from the sample and transmitted to a dichroic mirror (D1), which separates the 400 and $800 \mathrm{~nm}$ components and delivers them to separate New Focus 2007 auto-balanced photoreceivers (PD1 and PD2). Apertures (A1 and A2) are placed in front of the detectors to limit the amount of collected light. They are both set at a radius of $0.9 \mathrm{~mm}$. The distance from the collection lens (L1) to the detectors is about $50 \mathrm{~cm}$. A portion of the reference beam is separated by means of a second beamsplitter (BS2) and sent to a second dichroic mirror (D2). The two output components from this mirror are delivered to the reference ports of the photoreceivers. This serves to cancel power fluctuations at the two wavelengths.

The heterodyne signal at each wavelength, which results from the interference of the backscattered probe beam and the appropriate Doppler-shifted reference beam, is detected by the photoreceiver. Each signal is measured using a Stanford Research 830 dual phase lock-in amplifier array. The true magnitude of the heterodyne signal is displayed on an oscilloscope or recorded by a computer.

Each sample consists of a cuvette with a thin layer of clear gelatin about $3 \mathrm{~mm}$ thick, followed by a layer of polystyrene microspheres suspended in gelatin. The probe beam enters the sample through the first layer and is brought to a focus at the interface between the first and the second layers. The two layers have the same refractive index, which ensures that there is no reflective interface between them. Therefore, any heterodyne signal observed can be attributed solely to scattering from the microspheres.

The polystyrene microspheres range from 0.53 to $4.6 \mu \mathrm{m}$ in diameter and are obtained from Bangs Laboratories, Polysciences Inc. and Spherotech Inc. The volume concentration of microspheres within the second layer is maintained at $1.3 \%$ for all samples. Based on an illumination volume of about 850 $\mu \mathrm{m}^{3}$, as defined by the waist at the focus of the probe beam and its coherence length, the average number of microspheres illuminated ranges from 140 for the $0.53 \mu \mathrm{m}$ diameter microspheres to 0.2 for the $4.6 \mu \mathrm{m}$ diameter microspheres. The refractive index ratio of the microspheres to the gelatin is measured independently to be $1.19 \pm 0.01$. To account for attenuation and absorption of the probe beam in the first layer of gelatin, the results are normalized using heterodyne signals from a cuvette in which the second layer of gelatin is replaced by a mirror.

The amplitude of the measured heterodyne signal depends primarily on three factors: the number of microspheres illuminated, their positions within the probe beam, and the amount of backscattering they produce. The first two factors are undesirable in that they can potentially skew the experiment and mask the periodic structure of the light scattering spectrum. In the present study, we eliminated these contributions by carefully aligning the 400 and $800 \mathrm{~nm}$ beam components and employing approximately equal beam waists at the focus, as described above. This ensures that the beam components at both wavelengths illuminate the same region of the sample, and thus the same microspheres. Therefore, by taking the ratio of the signals at the two wavelengths, any dependence on the number and positions of the microspheres are normalized out.

\section{Theory}

In this section, we show that the heterodyne signal from multiple scatterers can be treated as an ensemble averaged heterodyne signal arising from a single scatterer. We then use this simplified theory in Sec. 4 to generate a computational model of our experiment.

The heterodyne signal resulting from the interference of a Doppler shifted reference beam and a signal beam, comprised of light scattered from multiple scatterers, can be derived by calculating the power of the total electric field and retaining only the frequency modulated term. In the case where the signal beam has a nonplanar wavefront, the result needs to be averaged over the finite size of the detector. The amplitude of the frequency modulated term can be expressed as:

$$
\begin{array}{r}
\left|\left[2 E_{r}(\vec{\rho}) E_{T}(\vec{\rho}) e^{i \psi_{T}(\vec{\rho})}\right]_{\vec{\rho}} \times e^{i \Delta \omega t}\right| \\
\quad=\left|\left[2 E_{r}(\vec{\rho}) E_{T}(\vec{\rho}) e^{i \psi_{T}(\vec{\rho})}\right]_{\vec{\rho}}\right|,
\end{array}
$$

where $E_{r}(\vec{\rho})$ is the electric field amplitude of the reference beam, $\vec{\rho}$ is the spatial coordinate on the detector's surface, $E_{T}(\vec{\rho})$ is the amplitude component of the signal electric field which is polarized in the same direction as the reference beam. (Henceforth, all discussion of electric fields refer to those with the same polarization as the reference beam.) The symbol, $\Delta \omega$, denotes the heterodyne frequency and $t$ represents the time. The associated phase shift of the signal field to the reference field is represented as $\psi_{T}(\vec{\rho})$. The bracket $[\ldots]_{\vec{\rho}}$ denotes averaging over the detector area and $[\ldots]$ denotes amplitude.

When the signal beam is comprised of the reflections from a group of scatterers, we can decompose it to the constituent electric fields from each scatterer. Accordingly, the heterodyne amplitude can be written as: 


$$
\begin{aligned}
& \left|\left[2 E_{r}(\vec{\rho}) E_{T}(\vec{\rho}) e^{i \psi_{T}(\vec{\rho})}\right]_{\vec{\rho}}\right| \\
& \quad=\left|\sum_{j=1}^{\infty} \sum_{i=1}^{\infty}\left[2 E_{r}(\vec{\rho}) E_{j, i}(\vec{\rho}) e^{i \psi_{j, i}(\vec{\rho})}\right]_{\vec{\rho}}\right|,
\end{aligned}
$$

where $E_{j, i}(\vec{\rho})$ denotes the amplitude of the scattered electric field associated with the $i$ th trajectory which has undergone exactly $j$ scattering events in the sample, and $\psi_{j, i}(\vec{\rho})$ is the associated phase.

Each term on the right hand side of Eq. (1b) can be rewritten as:

$$
\left[2 E_{r}(\vec{\rho}) E_{j, i}(\vec{\rho}) e^{i \psi_{j, i}(\vec{\rho})}\right]_{\vec{\rho}}=a_{j, i} \bar{E}_{r} \bar{E}_{j, i} e^{i \bar{\psi}_{j, i},}
$$

where $\bar{E}_{r}$ and $\bar{E}_{j, i}$ denote the root-mean-square averages of $E_{r}(\vec{\rho})$ and $E_{j, i}(\vec{\rho})$ over the detector areas, and $\bar{\psi}_{j, i}$ the effective average phase shift. The phase and amplitude of the scattered field must be averaged over the detector since light incident on scatterers will be scattered in different amounts at different angles. The reference field is assumed to have a Gaussian transverse spatial variation across the detector. The quantity $a_{j, i}$ is defined as the spatial coherence factor. ${ }^{10}$ It measures the uniformity of $\psi_{j, i}(\vec{\rho})$, as well as the correlation of $E_{j, i}(\vec{\rho})$ with $E_{r}(\vec{\rho})$. Its maximum value, 2, occurs when $\psi_{j, i}(\vec{\rho})$ is constant over the detector surface, and $E_{j, i}(\vec{\rho})$ is identical in profile to $E_{r}(\vec{\rho})$. Note that $\bar{E}_{r}$ and $\bar{E}_{j, i}$ are simply the square roots of the average reference intensity and scattered intensity at the detector, respectively. The choice of using the root-mean-square averages of $E_{r}(\vec{\rho})$ and $E_{j, i}(\vec{\rho})$ simplifies the final expression for the heterodyne signal amplitude.

Extensive discussion of $a_{j, i}$ and its behavior with respect to the number of scattering events, $j$, can be found in Ref. 10 . It is demonstrated in that reference, and indirectly in Refs. 7 and 11 , that $a_{j, i}$ decreases very rapidly with increasing values of $j$. This implies that a heterodyne measurement strongly favors the detection of singly scattered light over multiply scattered light, provided that the two are present in comparable amounts. Such is the case for light scattered near the surface of a turbid medium. Thus, in our case the measured heterodyne signal can be approximated by the terms associated with the $a_{1, i}$ 's in Eq. (1b):

$$
\left|\left[2 E_{r}(\vec{\rho}) E_{T}(\vec{\rho}) e^{i \psi_{T}(\vec{\rho})}\right]_{\vec{\rho}}\right| \approx \bar{E}_{r}\left|\sum_{i=1}^{N} a_{1, i} \bar{E}_{1, i} e^{i \bar{\psi}_{1, i}}\right| .
$$

Note that since the number of trajectories with only one scattering event equals the number of scatterers illuminated, $N$, the summation in Eq. (3) is terminated at $N$.

The heterodyne signal amplitude can be evaluated from Eq. (3) as

$$
\begin{aligned}
H & =\left|\left[2 E_{r}(\vec{\rho}) E_{T}(\vec{\rho}) e^{i \psi_{T}(\vec{\rho})}\right]_{\vec{\rho}}\right|, \\
& \approx \bar{E}_{r} \sqrt{\sum_{i=1}^{N}\left(a_{1, i} \bar{E}_{1, i}\right)^{2}+\sum_{i=1}^{N} \sum_{\substack{i^{\prime}=1 \\
i^{\prime} \neq i}}^{N} a_{1, i} a_{1, i^{\prime}} \bar{E}_{1, i} \bar{E}_{1, i^{\prime}} \cos \left(\bar{\psi}_{1, i}-\bar{\psi}_{1, i^{\prime}}\right)}, \\
& \approx \bar{E}_{r} \sqrt{\sum_{i=1}^{N}\left(a_{1, i} \bar{E}_{1, i}\right)^{2}} .
\end{aligned}
$$

The cross terms in Eq. (4) are negligible if the $\bar{\psi}_{j, i}$ 's are uncorrelated and the number of scatterers, $N$, in the probe region is large. We can also satisfy the later requirement by averaging over a sufficient number of measurements taken at different points on the sample. The assumption that the $\bar{\psi}_{j, i}$ 's are uncorrelated may be justified by noting that, in addition to the nonzero phase shift that the scattered light accumulates from the scatterers, there are additional random phase shifts due to the different round trip distances traversed by the light from scatterers at various depths. Given that the average spatial distance between neighboring scatterers in our experiment is at least $1.8 \mu \mathrm{m}$ or about 2.2 times the $800 \mathrm{~nm}$ wavelength, the scattered light contributions are unlikely to be correlated.

For a medium with microspheres of diameter $D$ illuminated by light of wavelength $\lambda$, we have an average detected field, $\bar{E}(\lambda, D)$, given by the square root of the average intensity of light per scatterer reaching the detector, $\bar{I}(\lambda, D)$. The root mean square ensemble average of the heterodyne signal amplitude from Eq. (4) can then be written as:

$$
\begin{aligned}
& \left.H(\lambda, D)\right|_{\text {ensemble-rms }} \\
& \quad=\left.\bar{E}_{r} \sqrt{\sum_{i=1}^{N}\left(a_{1, i}\left(\lambda, D, \vec{d}_{i}\right) \bar{E}_{1, i}\left(\lambda, D, \vec{d}_{i}\right)\right)^{2}}\right|_{\text {ensemble-rms }} \\
& \quad=\sqrt{N} \bar{E}_{r} \bar{a}(\lambda, D) \bar{E}(\lambda, D)=\sqrt{N} \bar{E}_{r} \bar{a}(\lambda, D) \sqrt{\bar{I}(\lambda, D)}
\end{aligned}
$$

with $\vec{d}_{i}$ the displacement of the $i$ th scatterer from the focal point. The parameter $\bar{a}(\lambda, D)$ is a defined quantity which accounts for the contributions of the $a_{1, i}\left(\lambda, D, \vec{d}_{i}\right)$ 's to the ensemble averaged heterodyne signal. As we shall see in Sec. 6, $\bar{a}(\lambda, D)$ is a fundamental characterization of the scattering process. Note that all quantities in Eq. (4) except $\bar{E}_{r}$ are functions of $\lambda, D$, and $\vec{d}_{i}$; we simply make these explicit in Eq. (5). The ensemble average is performed by summing over all possible $\vec{d}_{i}$.

In intensity-based LSS, the number of illuminated scatterers, $N$, can be found by measuring the backscattered intensity at many wavelengths. ${ }^{1}$ The same can be done in field-based LSS. Unfortunately, in the current feasibility demonstration, where the heterodyne signals are measured at only two wavelengths, $N$ cannot be accurately determined. However, since the beam components at the two wavelengths are well aligned, and thus illuminate the same focal area, the same scatterers are simultaneously illuminated at both wavelengths in each measurement. To insure that our data approaches the ensemble average, we make $M$ individual measurements of $H$, each at a different beam position. We then sum over the 
square of the measurements at each wavelength. The ratio, $R$, of the two sums then eliminates any dependence on $N$ :

$$
R=\frac{\sqrt{\sum_{l=1}^{M} H_{l}^{2}\left(\lambda_{1}, D\right)}}{\sqrt{\sum_{l=1}^{M} H_{l}^{2}\left(\lambda_{2}, D\right)}} \approx \frac{\bar{a}\left(\lambda_{1}, D\right) \sqrt{\bar{I}\left(\lambda_{1}, D\right)}}{\bar{a}\left(\lambda_{2}, D\right) \sqrt{\bar{I}\left(\lambda_{2}, D\right)}} .
$$

Note that in addition to the scattered intensity, $\bar{I}(\lambda, D)$, which determines the reflectance spectrum in a conventional LSS experiment, field-based LSS is also influenced by the wavelength-dependent spatial coherence factor, $\bar{a}(\lambda, D)$. This indicates that the field-based LSS spectrum will differ fundamentally from the intensity-based LSS spectrum.

\section{Computational Model}

The ensemble averaged quantity $\bar{a}(\lambda, D) \sqrt{\bar{I}(\lambda, D)}$ can be calculated from Mie scattering theory for spherical objects. We first express the contribution to the heterodyne signal amplitude from a single scatterer at a displacement $\vec{d}_{i}$ from the focal point. This is given by:

$$
H\left(\lambda, D, \vec{d}_{i}\right)=\left|\left[2 E_{r}(\lambda, \vec{\rho}) E_{i}\left(\lambda, \vec{d}_{i}\right) \frac{S\left(\lambda, D, \phi_{i}, \theta_{i}\right)}{k r}\right]_{\vec{\rho}}\right|,
$$

with $E_{i}\left(\lambda, \vec{d}_{i}\right)$ the electric field strength at the scatterer and $S\left(\lambda, D, \phi_{i}, \theta_{i}\right)$ the component of the scattered field (after it is collimated by the collection optics) with the same polarization as the reference beam. ${ }^{5}$ [Note that, despite its name, $S\left(\lambda, D, \phi_{i}, \theta_{i}\right)$ is a complex function.] In our notation, $\theta_{i}$ is the angle subtended from the vertical, defined as the direction of the probe beam's propagation, and $\phi_{i}$ is the angle in the plane normal to the vertical, with $\phi_{i}=0$ being along the direction of polarization. The angle of scattering, $\theta_{i}$ and $\phi_{i}$ can be related to $\vec{\rho}$ and $\vec{d}_{i}$ by Fourier optics, ${ }^{12} k$ is the optical wave number and $r$ the distance from the scatterer to the detector.

$S\left(\lambda, D, \phi_{i}, \theta_{i}\right)$ can, in turn, be expressed as:

$$
\begin{aligned}
S\left(\lambda, D, \phi_{i}, \theta_{i}\right)= & \sin ^{2}\left(\phi_{i}\right) S_{1}\left(\lambda, D, \theta_{i}\right) \\
& +\cos ^{2}\left(\phi_{i}\right) S_{2}\left(\lambda, D, \theta_{i}\right),
\end{aligned}
$$

where $S_{1}\left(\lambda, D, \theta_{i}\right)$ and $S_{2}\left(\lambda, D, \theta_{i}\right)$ are the amplitude functions given by Mie theory for scattering when the incident polarization is perpendicular and parallel to the scattering plane, respectively. ${ }^{13}$

From Eq. (5), the ensemble averaged quantity $\bar{a}(\lambda, D) \sqrt{\bar{I}(\lambda, D)}$ equals the root-mean-square ensemble average of the heterodyne signal associated with $N=1$. In other words, it can be expressed in terms of the single scatterer's heterodyne contribution:

$$
\begin{aligned}
\bar{a}(\lambda, D) \sqrt{\bar{I}(\lambda, D)} & =\frac{1}{\bar{E}_{r}} H\left(\lambda, D, \vec{d}_{i}\right)_{\text {ensemble-rms }} \\
& =\frac{1}{\bar{E}_{r} \sqrt{Q}} \sqrt{\sum_{\text {all } \bar{d}_{i}} H^{2}\left(\lambda, D, \vec{d}_{i}\right)},
\end{aligned}
$$

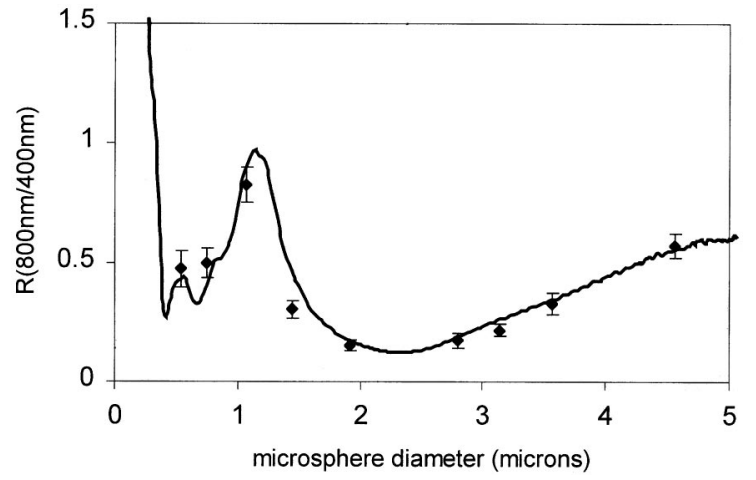

Fig. 2 Experimental data with theoretical fit (solid line).

where $Q$ is the number of elements in the ensemble average.

Using this formulation, we calculate the theoretical predictions for $R$ for microspheres ranging from 0.1 to $5 \mu \mathrm{m}$ in diameter. The result is then averaged over a $5 \%$ variation in diameter, to account for the distribution of microsphere sizes in a given sample. This distribution is consistent with the size distribution given by the manufacturers.

\section{Results}

The experimental data are plotted in Figure 2. Each data point consists of 30 or 45 measurements taken at various sample positions. We average over scatterer position by taking the root-mean-square of the measured heterodyne signal at each wavelength. The ratio of the results, $R$, is then calculated and plotted.

The solid line in the plot is the theoretical fit. The fit was done using the experimental specifications as listed in Sec. 2. In the fitting procedure, we used the aperture size of the detectors as free parameters. We found that the best fit requires that we use an aperture size of $0.7 \mathrm{~mm}$ in radius, instead of $0.9 \mathrm{~mm}$, for the $400 \mathrm{~nm}$ measurements. This can be accounted for as a possible misalignment of the incoming beam at the detector, which would result in a smaller effective detection area.

The theoretical fit agrees well with experiment for microspheres smaller than $5 \mu \mathrm{m}$ in diameter. However, the theory breaks down for the larger microsphere sizes. This is because the theoretical fit, based on Mie scattering theory, assumes an incident plane wave field. As the microsphere size becomes comparable to the beam waists (about $6 \mu \mathrm{m}$ in this experiment), this criterion is no longer satisfied.

\section{Discussion}

While both field-based and intensity-based LSS determine the size and relative refractive index of scatterers by measuring variations in scattering across the spectrum, field-based LSS differs from intensity-based LSS in two important ways. First, it is sensitive to phase front variations in the scattered wave, as well as to the intensity variations of the backscattered light. Second, it permits greater localization of the region to be measured.

Single particle light scattering is characterized by the scattering amplitude, $S(\lambda, D, \phi, \theta)$, a complex function with a phase that varies with angular coordinates. This phase is not 
measured in intensity-based LSS. In contrast, field-based LSS is very sensitive to variations in phase. To illustrate this, we consider an experiment with a plane wave incident field. The average scattering intensity from a single scatterer measured at the detector can then be expressed as: ${ }^{5}$

$$
\bar{I}(\lambda, D)=\frac{\bar{E}_{i}^{2}}{k^{2} r^{2}}\left[|S(\lambda, D, \phi, \theta)|^{2}\right]_{\rho} .
$$

From Eq. (7), a similar field-based LSS experiment in which the incident and reference fields are both plane waves will give a heterodyne signal of the form:

$$
H(\lambda, D)^{2}=\frac{4 \bar{E}_{r}^{2} \bar{E}_{i}^{2}}{k^{2} r^{2}}|[S(\lambda, D, \phi, \theta)] \vec{\rho}|^{2}
$$

Setting aside the average reference field intensity, $\bar{E}_{r}^{2}$, a subtle but important difference between these two expressions can be seen. In Eq. (10), the magnitude of $S(\lambda, D, \phi, \theta)$ is taken before averaging over the detector area, whereas in Eq. (11), the sequence of operations is reversed. Thus, phase variations become important in field-based LSS, and the measured signal will be proportional to the degree of phase-front uniformity of the scattered light. More explicitly, the measured signal strength depends on how strongly the phase [argument of $S(\lambda, D, \phi, \theta)]$ varies with $\theta$. We found, from Mie theory, that this phase, like the magnitude of $S(\lambda, D, \phi, \theta)$, has a stronger angular dependence for larger ratio $(D / \lambda)$. This additional dependence on $(D / \lambda)$ should lead to more variation across the spectrum with field-based LSS than with intensity-based LSS. This, in turn, should make calculation of scatterer size easier and more sensitive.

The theory presented in Sec. 3 employs an ensembleaveraged spatial coherence factor, $\bar{a}(\lambda, D)$, to quantify the degree of phase front uniformity. We note that $\bar{a}(\lambda, D)$ is fundamentally related to $S(\lambda, D, \phi, \theta)$. By substituting Eqs. (10) and (11) into Eq. (5) and setting $N=1$ (as the above calculation is for a single scatterer), we obtain:

$$
\bar{a}(\lambda, D)=2 \sqrt{\frac{\left|[S(\lambda, D, \phi, \theta)]_{\rho}\right|^{2}}{\left[|S(\lambda, D, \phi, \theta)|^{2}\right] \vec{\rho}}} .
$$

[Footnote: Eq. (12) is applicable only in situations where both the reference and incident fields are uniform, and must be appropriately modified for nonuniform input fields.]

To clarify the physical significance of $\bar{a}(\lambda, D)$, we express Eqs. (10) and (11) in terms of the scattering cross section, $\sigma(\lambda, D)$, and phase function, $f(\lambda, D, \phi, \theta)$. The phase function is the normalized function which describes the angular intensity distribution of the scattered light, ${ }^{14}$ given by Ref. 5 :

$$
f(\lambda, D, \phi, \theta)=\frac{1}{k^{2} \sigma(\lambda, D)}|S(\lambda, D, \phi, \theta)|^{2} .
$$

We can thus express Eq. (10) as

$$
\bar{I}(\lambda, D)=\frac{\bar{E}_{i}^{2}}{r^{2}} \sigma(\lambda, D)[f(\lambda, D, \phi, \theta)] \vec{\rho}
$$

The corresponding equation for the heterodyne signal, Eq. (11), can then be written in terms of $\bar{a}(\lambda, D)$ as:

$$
H(\lambda, D)^{2}=\frac{\bar{E}_{r}^{2} \bar{E}_{i}^{2}}{r^{2}} \sigma(\lambda, D) \bar{a}^{2}(\lambda, D)[f(\lambda, D, \phi, \theta)]_{\vec{\rho}} .
$$

In intensity-based light scattering measurements, the scattering cross-section, $\sigma(\lambda, D),{ }^{14}$ and the anisotropy factor, $g(\lambda, D),{ }^{14}$ are often used to characterize the scattering process. In contrast, specification of the scattering process in field-based light scattering requires a different parameter, the spatial coherence factor $\bar{a}(\lambda, D)$, derived from $S(\lambda, D, \phi, \theta)$.

In addition to this sensitivity to phase variations, fieldbased LSS has the advantage of being able to localize a very small region for study. This localization can be achieved with low-coherence interferometric techniques, as exemplified by OCT. $^{7-9}$ Spatial localization is limited by the coherence length, and a typical femtosecond laser beam can localize a region for study on the order of tens of microns. This opens the possibility of selectively probing each individual scatterer, such as a single cell nucleus, even when it is surrounded by other scatterers. The prospect of probing one scatterer at a time also simplifies the computations. For a single scatterer, the cross-terms in Eq. (4) will not be present, thereby eliminating the need to average over numerous samples. We also note that with the high sensitivity afforded by heterodyne techniques, the full-spectral response of field-based LSS may provide a means to resolve features of a scatterer that are considerably smaller than an optical wavelength.

\section{Conclusion}

This study demonstrates the feasibility of field-based LSS. We have shown that measurements taken with this technique agree with our theoretical modeling of the scattering and propagation of the scattered field. According to the theory, the field-based technique is sensitive to phase-front variations, which we have parametrized as $\bar{a}(\lambda, D)$. This factor is fundamentally related to the scattering function. The use of low coherence interferometry in field-based LSS also implies that functional information about the target region can be mapped in three dimensions.

The next step of this study will be a full implementation of this field-based LSS technique in which measurements will be made over a number of wavelengths. From this set of spectral response data, we will be able to uniquely evaluate the size of spherical scatterers, such as cell nuclei. This will provide a useful tool for in vivo diagnosis of precancerous changes in the epithelium.

\section{Acknowledgments}

Valuable discussions with Vadim Backman are gratefully acknowledged, as well as help by Rajan Gurjar with various aspects of the experiment. This work was carried out at the MIT Laser Biomedical Research Center and was supported by National Institutes of Health Grant No. P41-RR02594 and a grant from Hamamatsu Corporation. 


\section{References}

1. L. T. Perelman, V. Backman, M. Wallace, G. Zonios, R. Manohaan, A. Nusrat, S. Shields, M. Seiler, C. Lima, T. Hamamo, I. Itzkan, J. Van Dam, J. M. Crawford, and M. S. Feld, "Observation of periodic fine structure in reflectance from biological tissue: A new technique for measuring nuclear size distribution,' Phys. Rev. Lett. 80, 627630 (1998).

2. V. Backman, R. Gurjar, K. Badizadegan, I. Itzkan, R. R. Dasari, L. T. Perelman, and M. S. Feld, "Polarized light scattering spectroscopy for quantitative measurement of epithelial cellular structures in situ," IEEE J. Sel. Top. Quantum Electron. 5(4), 1019-1026 (1999).

3. V. Backman, R. Gurjar, K. Badizadegan, G. Zonios, I. Itzkan, R. R. Dasari, J. M. Crawford, J. Van Dam, L. T. Perelman, and M. S. Feld, "Light scattering spectroscopy for early cancer diagnosis," Proceedings of ICOLS99, 1999 (unpublished).

4. L. T. Perelman, G. Zonios, V. Backman, R. Gurjar, R. R. Dasari, I. Itzkan, and M. S. Feld, "Quantitative analysis of mucosal tissues in patients using light scattering spectroscopy, optical tomography and spectroscopy of tissue III,' Proc. SPIE 3597, 474-479 (1999).

5. V. de Hulst, Light Scattering by Small Particles, Dover Publications, Inc., New York (1957).

6. R. S. Cotran, S. L. Robbins, and V. Kumar, Robbins Pathological
Basis of Disease, W. B. Saunders Company, Philadelphia (1994).

7. M. R. Hee, J. A. Izatt, J. M. Jacobson, J. G. Fujimoto, and E. A. Swanson, "Femtosecond transillumination optical coherence tomography," Opt. Lett. 18, 950-952 (1993).

8. J. A. Izatt, M. R. Hee, G. Owen, E. A. Swanson, and J. G. Fujimoto, "Optical coherence microscopy in scattering media," Opt. Lett. 19, 590-592 (1994).

9. J. A. Izatt, M. D. Kulkarni, H. Wang, K. Kobayashi, and M. V. Sivak, "Optical coherence tomography and microscopy in gastrointestinal tissues," IEEE J. Sel. Top. Quantum Electron. 2, 1017-1028 (1996).

10. C. Yang, K. An, L. T. Perelman, R. R. Dasari, and M. S. Feld, "Spatial coherence of forward scattered light in a turbid medium," $J$. Opt. Soc. Am. A 16, 866-871 (1999).

11. A. Wax and J. E. Thomas, "Measurement of smoothed Wigner phase-space distributions for small-angle scattering in a turbid medium," J. Opt. Soc. Am. A 15, 1896-1908 (1998).

12. J. W. Goodman, Introduction to Fourier Optics, McGraw-Hill, San Francisco (1968)

13. C. Bohren and D. Huffman, Absorption and Scattering of Light by Small Particles, pp. 61-63, Wiley-Interscience, New York (1983).

14. A. Ishimaru, Wave Propagation and Scattering in Random Media, Vol. 2, Academic, New York (1978). 